\title{
El hundimiento de la evaluación online: una perspectiva de la situación actual
}

\author{
The collapse of online evaluation: an overview of the current situation
}

\author{
Víctor González Calatayud \\ Universidad Miguel Hernández de Elche (España) \\ victor.gonzalezc@umh.es
}

No cabe duda de que el año 2020 será reconocido como uno de los años en los que mayor impulso ha tenido la enseñanza online, todo derivado de la pandemia mundial del SARS-CoV-2. Los centros educativos de todos los niveles cerraron y los profesores tuvieron que ponerse manos a la obra para trabajar con sus alumnos de manera virtual. Muchos de estos profesionales tenían poca o nula experiencia con el uso de herramientas para la enseñanza a distancia. Pero, no cabe duda de que el problema no ha sido únicamente la poca formación del profesorado en relación con la Tecnología Educativa. Esta situación ha puesto en relieve uno de los grandes problemas de la educación: entender la evaluación como un proceso en el que se comprueba el mero logro de un conocimiento teórico. Se juntan, por tanto, dos problemáticas: un mal entendimiento del proceso evaluativo y docentes que no saben cómo actuar en situaciones de enseñanza online.

El proceso evaluativo debe ser entendido como sistema orientado al aprendizaje, en el que la retroalimentación sea un elemento esencial que permita la mejora del aprendizaje continuo (García-Peñalvo, Corell, Abella-García y Grande, 2020). Así, el concepto de evaluación no puede ser entendido únicamente como un proceso de medición o calificación del alumnado, sino que el proceso evaluativo debe incluir ambos y proporcionar algo más al proceso de aprendizaje. No consiste solo en proporcionar una nota, sino un proceso de diálogo, comprensión y mejora que revierta positivamente en el proceso de enseñanza y aprendizaje (Santos Guerra, 1998). Como recogen Castillo y Cabrerizo (2009) y GarcíaPeñalvo et al. (2020), la calificación es el sistema por el que se realiza una valoración o una medición para expresar el grado de suficiencia en relación con los conocimientos, destrezas y habilidades de los estudiantes a partir del uso de un instrumento de evaluación -examen, actividad o prueba-. Por lo que ceñirse únicamente a medir o calificar no permite que el alumnado pueda mejorar en su proceso de aprendizaje. Sin embargo esta premisa no es concebida por una gran porcentaje del profesorado (Freitas, 2012).

Y si nos centramos en el ámbito de lo virtual la evaluación se vuelve un elemento esencial a tener en cuenta. No podemos obviar que el alumnado que trabaja de manera virtual siente un cierto desamparo y le falta el contacto con el profesor mientras realiza las tareas académicas. Así que, si consideramos el proceso evaluativo como un sistema de mejora no podemos caer en la tentación de únicamente ceñirnos a una evaluación sumativa basada exclusivamente en la calificación de las tareas, exámenes o pruebas que le pongamos al alumnado. El alumnado que trabaja de manera online espera una pronta respuesta además de que el propio proceso requiere de ese feedback que le pueda dar el docente. Y es que la enseñanza online y la evaluación correspondiente tiene una serie de mitos que la gente que no ha cursado este tipo de enseñanza suele repetir de forma constante.

El primero y más destacable en el momento actual es el lema de "en la enseñanza online es más fácil copiar". Una máxima para los alumnos que en estas fechas se están examinando de manera online en centros de estudios que normalmente se trabaja de manera presencial. Touron (2015) indicaba que una de las principales preocupaciones de los educadores en la enseñanza online era evitar que se copien los alumnos. Eso sí, justifica este mito indicando que las plataformas suelen incorporar sistemas que evitan el fraude. Pero me gustaría ir un paso 
más allá. Y es que si la concepción sobre el proceso evaluativo cambia, el que los alumnos se copien se vuelve imposible. Si pensamos que el examen o prueba que se prepara para el alumnado se basa principalmente en la reproducción de contenido el alumnado siempre encontrará el método más conveniente para copiarse. Pero si nos centramos en una evaluación de las competencias todo cambia.

Si entendemos la competencia como la aptitud o capacidad para movilizar rápidamente y de manera eficaz recursos, conocimientos, habilidades y actitudes que nos lleven a afrontar situaciones de manera adecuada (Cano, 2018), la evaluación irá enfocada a comprobar que una persona responde de manera adecuada ante cierta situación. O lo que es lo mismo, la evaluación se centrará en comprobar que el alumnado tiene unos conocimientos que sabe aplicar, adaptar y reutilizar en función de la situación dada.

Otro de los grandes mitos que la evaluación online suele tener es pensar que la única o mejor manera de evaluar al alumnado en esta modalidad es a través de pruebas objetivas de elección múltiple. $Y$ esto no solo ocurre a nivel online. Si se echa un vistazo por las guías docentes de las asignaturas de la mayoría de las titulaciones de las universidades, este es el instrumento fundamental de evaluación de los conocimientos del alumnado (Dorrego, 2006). Si, como decíamos anteriormente, la evaluación debe ser considerada como un instrumento de mejora del aprendizaje, es fundamental que el proceso de diseño de la evaluación online se base en un enfoque constructivista y holístico que se centre en una evaluación formativa del aprendizaje. En primer lugar, se requiere de un diseño instruccional que se centre en el aprendizaje del alumnado, y, en segundo lugar, partir de ese objetivo para diseñar una evaluación que utilice distintos métodos y se lleve a cabo en distintos momentos con la intención de que el alumnado aprenda.

Una vez tengamos claro el proceso de evaluación, hay diversos instrumentos que podemos utilizar para la evaluación, ya sea al inicio, durante o final del curso. A parte de las pruebas objetivas, podemos desarrollar pruebas adaptativas, es decir pruebas individualizadas en función de las habilidades del estudiante. También podemos pedir el desarrollo de un proyecto colaborativo o la resolución de problemas en grupo. Se puede, incluso, utilizar las redes sociales como instrumento de heteroevaluación donde expertos de diversa índole colaboren en la evaluación. Igualmente, existen herramientas que nos permiten introducir estrategias del juego en la evaluación como son kahoot, Quizizz o flipgrip. Esta última nos permite realizar una pregunta y que los alumnos contesten mediante vídeos.

Pero no solo nos debemos quedar en el instrumento dentro de la evaluación, sino que los criterios y su relación con las competencias a evaluar se convierten en algo fundamental. El alumnado que está cursando enseñanza a distancia requiere de información constante de su proceso de aprendizaje. Y para ello las rúbricas se convierten en un elemento esencial dentro del proceso de trabajo. Tener un sistema en el que el alumnado se pueda basar de forma constante para el desarrollo de las tareas, actividades, pruebas y diversas situaciones a las que se enfrenta es esencial para su proceso de aprendizaje. Se trata de una forma de autoevaluación.

Así, otro de los mitos que se le suelen atribuir a la enseñanza online y, por tanto, a la evaluación online es que se trata de un proceso individual. No suele entrar en la preparación de cursos online la realización de actividades colaborativas entre los estudiantes y, muchos menos, de situaciones de evaluación. Pero no podemos olvidar que la colaboración online favorece el aprendizaje de otro tipo de competencias que de manera individual no se pueden adquirir. Competencias que están integradas en las competencias genéricas de las titulaciones y que muchas veces se olvidan trabajar y evaluar. De este modo, en cuanto a evaluación online se refiere, la realización de tareas coevaluativas puede ser fundamental para el desarrollo de ciertas competencias.

Por último, me gustaría destacar la realización de procesos innovadores de evaluación online. Cada vez más podemos observar como docentes integran elementos de ludificación a la hora de evaluar (Falcó-Boudet y Huertas, 2018). La gamificación o la incorporación de metodologías basadas en el juego es uno de los muchos métodos que podemos integrar para mejorar la evaluación continua del alumnado. Pero no solo nos debemos quedar ahí. Sino que la incorporación de métodos de evaluación centrados en el alumnado y que se basen en la 
personalización deberían ser cada vez más recurrentes, tanto a nivel presencial como online. Y para ello, la Tecnología Educativa tiene mucho que decir, sobre todo en la enseñanza virtual. No podemos quedarnos impresionados por el uso de herramientas que tenemos a nuestra disposición. Debemos considerar siempre, en primer lugar, todo el proceso de planificación, desde los objetivos hasta la evaluación. Seguir una misma línea entre los objetivos que proponemos, la metodología que vamos a utilizar y el proceso de evaluación -inicial, continuo y final- que se pretende usar. La evaluación en un sistema online no puede quedar, como está sucediendo, en una mera prueba objetiva.

En este número 8 de la revista RiiTE contamos con una gran diversidad de artículos centrados en diversos ámbitos, incluyendo estudios centrados en población adolescente, universitaria y con necesidades educativas especiales. El primer artículo realizado por Patricia Abelairas-Etxebarria y Jon Mentxaka de la Universidad del País Vasco nos ofrecen un estudio sobre lo que opinan los estudiantes sobre el uso académico de las redes sociales, siendo una de las principales conclusiones que los estudiantes están dispuestos a introducirlas como herramienta en su formación universitaria. También en relación con las redes sociales, Cristina Sánchez de la UNED y $M^{a}$ Carmen López nos presentan la percepción de actitudes nocivas en el uso de las redes sociales en jóvenes adolescentes.

Otro de los artículos incluidos en este número es realizado por Nancy Castillo y José Jiménez de la Universidad Rovira i Virgili. En este artículo presentan un trabajo en el que implementaron material educativo gamificado para enseñar matemáticas a los alumnos con Síndrome de Down. En otra línea, Daniel Romero y Bárbara de Benito de la Universidad de las Islas Baleares han realizado un trabajo relacionado con el uso de simuladores virtuales para la enseñanza en la rama sanitaria dentro de la Formación profesional.

Emma María Ortega y su equipo presentan un trabajo centrado en el aprendizaje del equilibrio químico a través de la combinación de software libre y técnicas analíticas de tal forma que los alumnos mejoren su comprensión. Entre los principales hallazgos está que se redujo el tiempo del proceso de aprendizaje. Por su parte, María del Pilar Ruiz, del CRA de Castilla-La Mancha, nos presenta el nivel de competencia digital docente del profesorado de las escuelas rurales de Albacete, siendo el uso que hacen de la tecnología limitado. Por último, Alberto Jiménez de la Universidad de Murcia ha realizado un trabajo en el que hace un análisis descriptivo de los Entornos Personales de Aprendizaje en la Enseñanza Obligatoria.

\section{REFERENCIAS BIBLIOGRÁFICAS}

Cano, E. (2018). La evaluación de las competencias genéricas. En E. Cano, J. Fabregat y J. Oliver (Coord.), La evaluación formativa y acreditativa de competencias. Competencias genéricas en la universidad Recursos en línea para entenderlas y aplicarlas (pp. 69.90). Barcelona: Transmedia XXI.

Castillo, S. y Cabrerizo, J. (2009). Evaluación educativa de aprendizajes y competencias. Madrid: Pearson.

Dorrego, E. (2006). Educación a distancia y evaluación del aprendizaje. RED, Revista de Educación a Distancia, 6, 1-23. https://www.um.es/ead/red/M6/dorrego.pdf

Falcó-Boudet, J.M. y Huertas, J.L. (2018). Superpoderes contra el Dr. Discriminador: La mejora de la evaluación continua mediante la ludificación en el Máster en profesorado. Revista interuniversitaria de investigación en Tecnología Educativa, 4, 68-81. http://dx.doi.org/10.6018/riite/2018/327961

Freitas, A. (2012). Estilos de enseñanza del profesorado de educación superior: estudio comparativo España-Brasil (Tesis Doctoral). Valladolid: Universidad de Valladolid

García-Peñalvo, F.J., Corell, A., Abella-García, V. y Grande, M. (2020). La evaluación online en la educación superior en tiempos de la COVID-19. Education in the Knowledge Society, 21, 1-26. http://doi.org/10.14201/eks.23013 
Santos Guerra, M.A. (1998). Evaluar es comprender. Buenos Aires: Ediciones Magisterio del Río de la Plata.

\section{INFORMACIÓN SOBRE EL AUTOR}

\section{Víctor González Calatayud}

Universidad Miguel Hernández de Elche

Profesor Ayudante doctor en el área de Didáctica y Organización Escolar de la UMH. Es Doctor en Tecnología Educativa por la Universidad de Murcia, hizo su tesis sobre ciberacoso. Es Licenciado en Pedagogía y tiene el Máster en Psicología de la Educación. Anteriormente fue profesor asociado en la Universidad de Murcia. Ha participado y participa en diversos proyectos nacionales e internacionales relacionados con las TIC, las NEE y la competencia de emprendimiento. Ha realizado estancias de investigación en la Universidad de Helsinki y en la Dublin City University.$$
\text { (c) (1) (2) }
$$

Los textos publicados en esta revista están sujetos a una licencia de Reconocimiento 4.0 España de Creative Commons. Puede copiarlos, distribuirlos, comunicarlos públicamente y hacer obras derivadas siempre que reconozca los créditos de las obras (autoría, nombre de la revista, institución editora) de la manera especificada por los autores o por la revista. La licencia completa se puede consultar en:Licencia Creative Commons Atribución-NoComercial-Compartir por igual 4.0 Internacional.
} 